\title{
Obstrucción intestinal por hernia obturatriz: Serie de casos
}

\author{
Juan F. Allamand T. ${ }^{1}$, Karen Schönffeldt G. ${ }^{3}$, José M. Campero M. ${ }^{3}$, \\ Consuelo Santibáñez P. ${ }^{3}$ y Nicolás Rojas F. ${ }^{2}$
}

\section{Intestinal obstruction caused by obturator hernia: Case series}

Objective: 3 cases of intestinal obstruction that resulted in diagnosis of obturator hernia, their management and a brief international literature review are presented. Material and methods: Discussion of the presentation, images, and management. In all cases surgery was performed the same day from the diagnosis of intestinal obstruction and confirmation of obturator hernia. The management of the hernia and it's content was adjusted to each patient's situation. Results: Our 3 patients, when reviewed retrospectively, had the classic presentations and images of obturator hernia. All of them had a favourable evolution with an early discharge and no complications on follow up. Discussion: Our 3 cases had clinical presentations and imaging studies that correlated well with international literature. Conclusion: Obturator hernia is an infrequent pathology, but appears in a particular type of patient and with classic presentation. Axial computed tomography is an important tool and usually makes the diagnosis previously to surgical exploration. It is important to consider this diagnosis when being faced with patients with intestinal obstruction.

Key words: intestinal obstruction; obturator; hernia.

\section{Resumen}

Objetivo: Se presentan 3 casos de obstrucción intestinal por hernia obturatriz atascada, su manejo y una breve revisión de la literatura. Materiales y Métodos: Discusión del cuadro clínico, imágenes y manejo. Todos los casos fueron operados dentro del mismo día del diagnóstico y se confirmó la presencia de hernia obturatriz. El manejo de la hernia y su contenido fue ajustado a la situación de cada paciente. Resultados: Los tres casos de hernia obturatriz aquí discutidos tienen la presentación clásica del cuadro, cuando se los revisa en forma retrospectiva y con imagenología compatible. Los tres pacientes evolucionaron en forma satisfactoria con alta precoz y control posoperatorio sin incidentes. Discusión: Nuestros 3 casos se condicen tanto en hallazgos clínicos, imagenológicos y en manejo con lo descrito en la literatura internacional. Conclusión: La hernia del agujero obturatriz siendo infrecuente, se presenta en un tipo particular de pacientes y con sintomatología clásica. La tomografía axial computada es de gran ayuda y suele hacer el diagnóstico preciso en el preoperatorio. Es importante recordar este diagnóstico diferencial al momento de estudiar pacientes con obstrucción intestinal.

Palabras clave: obstrucción intestinal; obturatriz; hernia.
Servicio de Emergencias Adulto, Hospital Padre Hurtado.

${ }^{2}$ Servicio de Cirugía, Hospital Padre Hurtado.

${ }^{3}$ Facultad de Medicina Clínica Alemana-Universidad del Desarrollo.

Recibido el 29 de abril de 2018 y aceptado para publicación el 5 de junio de 2018.

Correspondencia a: Dr. Juan F. Allamand T. juanfcoallamand@gmail.com

\section{Introducción}

La hernia obturatriz es la protrusión patológica de contenido abdominal a través del canal obturatriz. Es una entidad infrecuente, representa menos del 1\% de las hernias de la pared abdominal y habitualmente se presentan como obstrucción intestinal ${ }^{1-3}$. Por su baja frecuencia y cuadro clínico poco conocido, suele ser de diagnóstico tardío, y muchas veces intraoperatorio ${ }^{3}$. A pesar del cuadro clínico y el tipo de paciente clásico, muchas veces es necesario el uso de imágenes para establecer el diagnóstico en el preoperatorio ${ }^{3}$. Tiene la mortalidad más alta reportada dentro de las hernias $(70 \%)^{1,2}$. Alta morbilidad asociada $(75 \%)^{1,2}$. Están descritos tanto manejos abiertos como laparoscópicos, cada uno con sus beneficios y desventajas ${ }^{2}$. 


\section{CASOS CLÍNICOS}

\section{Materiales y Métodos}

A continuación se presentan casos de obstrucción intestinal con diagnóstico de hernia obturatriz que comprende el período entre 2012-2018 en el Servicio de Urgencias del Hospital Padre Hurtado. Se describen la presentación clínica, los hallazgos imagenológicos, el manejo y la evolución posterior. Se obtuvo consentimiento informado para la realización de esta investigación.

\section{Caso 1}

Mujer de 80 años, con antecedentes de hipertensión y ataque cerebrovascular previo, consulta por dolor abdominal, vómitos y ausencia de expulsión de gases de un día de evolución. Al examen físico destaca, dolor y masa palpable indurada en región crural derecha. Dado duda diagnóstica, se realiza tomografía computarizada (TC) la cual se informa como hernia femoral (Figuras 1 y 2). Se realiza abordaje preperitoneal de Nyhus, donde se halla hernia obturatriz derecha con asa necrótica de íleon terminal en su interior. Se accede a cavidad peritoneal, y se realiza resección y anastomosis manual de segmento de $15 \mathrm{~cm}$ Se repara defecto de pared con plug de malla de polipropileno. Alta precoz, sin incidentes.

\section{Caso 2}

Mujer de 91 años, con antecedentes de fibrilación auricular paroxística y síndrome consuntivo, consulta por cuadro de 3 días de evolución de dolor abdominal difuso asociado a vómitos oscuros y de mal olor. Al examen físico destaca abdomen distendido, ruidos hidroaéreos disminuidos y dolor a la palpación difusa, sin signos de irritación peritoneal y con ampolla rectal vacía. Se realiza TC que muestra obstrucción intestinal (Figuras 3 y 4), pero que inicialmente no logra concluir en una etiología clara. En pabellón, se realiza laparotomía media infraumbilical con hallazgo de hernia obturatriz derecha atascada, con un segmento de íleon distal

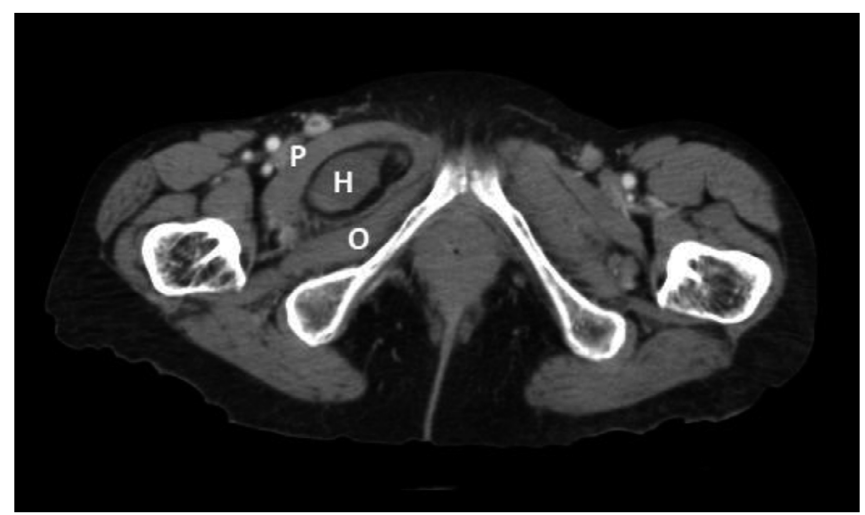

Figura 1. Tomografía axial computarizada perteneciente a paciente del caso 1, corte axial. P: Músculo pectíneo. H: Hernia. O: Músculo obturador.

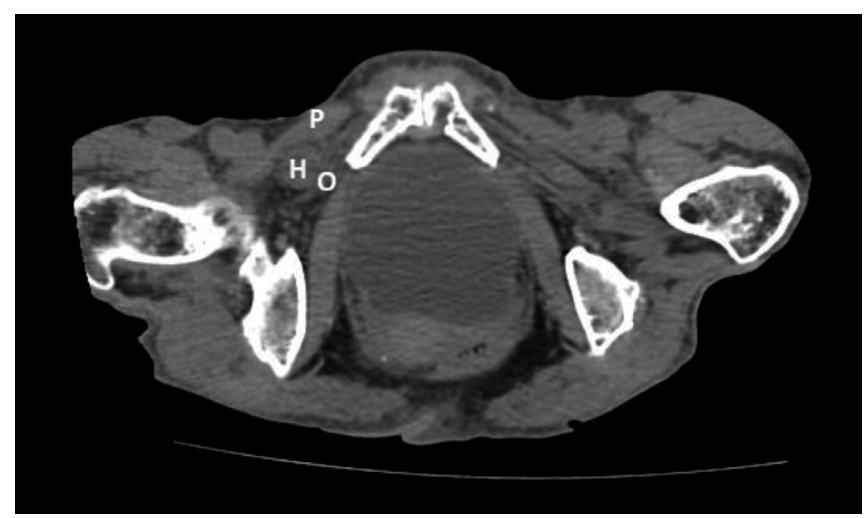

Figura 3. Tomografía axial computarizada perteneciente a paciente del caso 2, corte axial. P: Músculo pectíneo. H: Hernia. O: Músculo obturador.

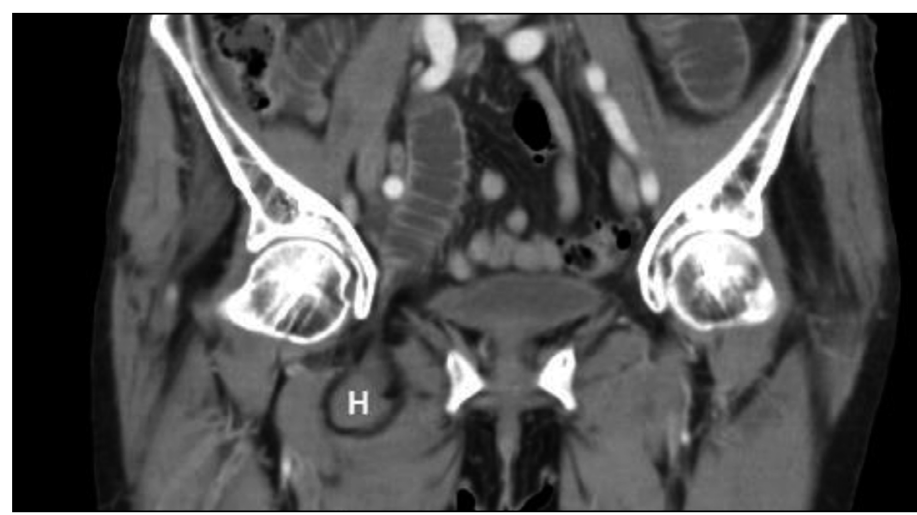

Figura 2. Tomografía axial computarizada perteneciente a paciente del caso 1 . H: Hernia.

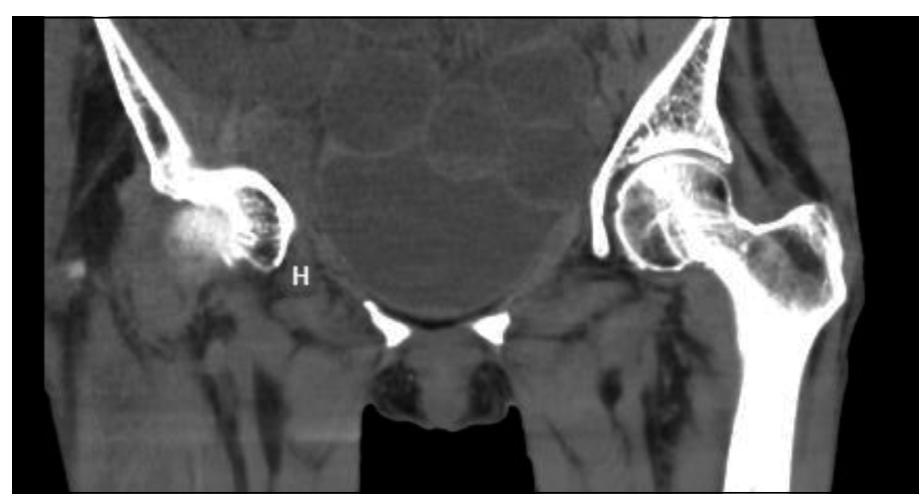

Figura 4. Tomografía axial computarizada perteneciente a paciente del caso 2. H: Hernia. 


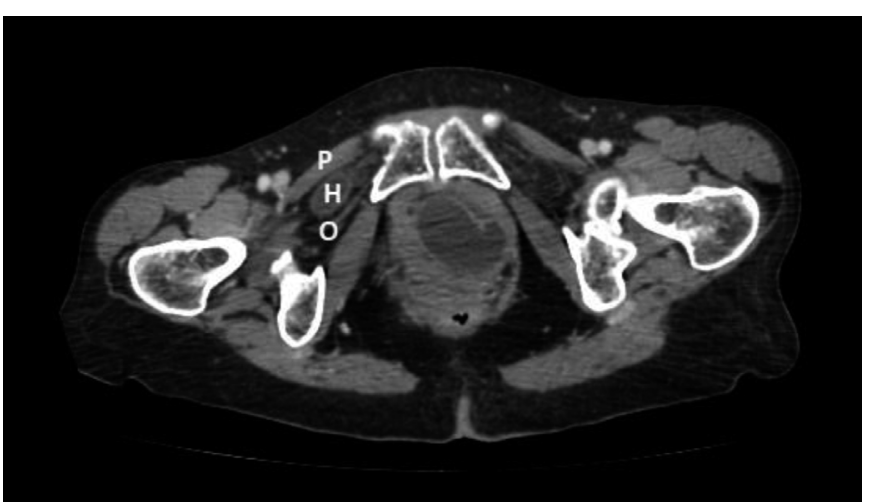

Figura 5. Tomografía axial computarizada perteneciente a paciente del caso 3, corte axial. P: Músculo pectíneo. H: Hernia. O: Músculo obturador.

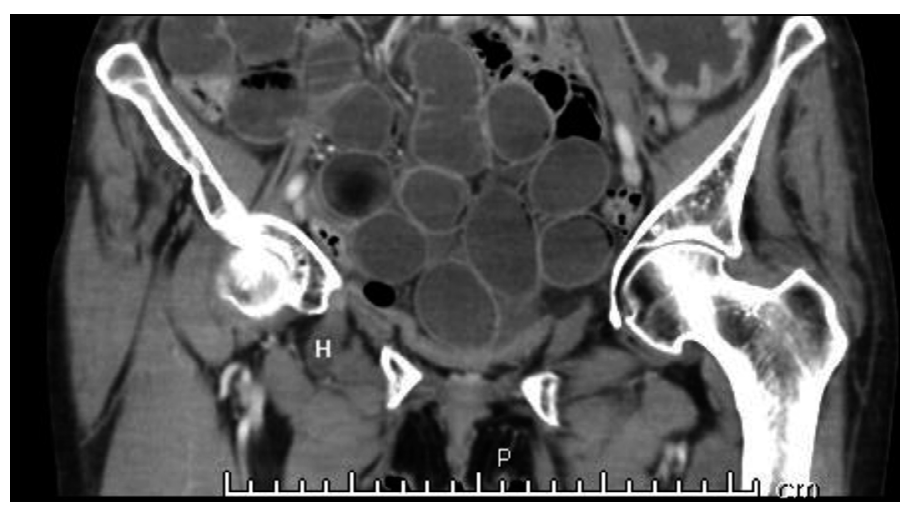

Figura 6. Tomografía axial computarizada perteneciente a paciente del caso 2. H: Hernia. estrangulado. Se reseca segmento de $5 \mathrm{~cm}$ con anastomosis manual y se repara el defecto herniario con punto simple de polipropileno. Inicialmente evoluciona séptica, con fibrilación auricular con rápida respuesta ventricular, lo cual se controla y logra el alta médica sin complicaciones a los pocos días.

\section{Caso 3}

Mujer de 78 años, con antecedentes de hipertensión, consulta por cuadro de dolor en muslo derecho gatillado por actividad, irradiado a abdomen inferior $\mathrm{y}$ asociado a náuseas y vómitos. $\mathrm{Al}$ examen físico destaca dolor a la palpación de hemiabdomen derecho. TC se informa como obstrucción intestinal por hernia femoral derecha (Figuras 5 y 6). Se opera con abordaje preperitoneal donde destaca pared abdominal delgada y se evidencia una hernia obturatriz derecha atascada con borde antimesentérico del íleon (tipo Richter). Se reduce, y se repara defecto con plug de malla de polipropileno. Paciente presenta cuadro de arritmia posterior a la cirugía que responde a terapia médica y posteriormente es dada de alta en buenas condiciones.

\section{Discusión}

\section{Definición y etiopatogenia}

Descrita en 1724 por Arnaud de Ronsil, el primer reporte de cirugía fue por Henry Obre en $1851^{1}$. Estas se dan en el canal del mismo nombre que contiene el nervio obturatriz y los vasos obturatrices. La debilitación de la membrana obturatriz, sumado a la disminución del tejido adiposo y linfático preperitoneal (corpus adiposum) circundante, facilita la formación hernia cuyo saco se ubica en la profundidad del muslo, entre el músculo pectíneo y el aductor largo ${ }^{1}$.

\section{Epidemiología}

Son cuadros infrecuentes. Reportes previos la citan en el $0,07 \%$ a $1 \%$ de todas las hernias, y hasta $0,2-1,6 \%$ de las obstrucciones intestinales bajas mecánicas ${ }^{1-4}$. Interesantemente, la mayor parte de las series reportadas son asiáticas: $0,073 \%$ de todas las hernias en Occidente, $1 \%$ en Oriente ${ }^{4}$. En la literatura inglesa se le conoce como "little old lady hernia" (hernia de pequeña señora añosa) ya que característicamente afectan a mujeres octogenarias $(85 \%)$, delgadas y a derecha ${ }^{1,2,5}$. Una reciente serie japonesa destaca con 13 pacientes, todas mujeres, con promedio de 78 años y con un IMC de $16,8^{6}$. Liu et al presentó 10 años después una serie de 10 pacientes con similares características ${ }^{7}$. Es posible que esta mayor incidencia en Oriente tenga relación con la fisionomía y longevidad.

\section{Clínica}

La gran mayoría de los casos (80-90\%) se presentan como cuadro de obstrucción ${ }^{1,8}$. Es frecuente el dolor irradiado en el muslo por medial hasta la rodilla, por afectación del nervio homónimo (Signo de Howship-Romberg) presente en el $15-50 \%{ }^{7,8}$. El $50 \%$ tiene sólo dolor abdominal bajo inespecífico. A su vez, el $60 \%$ tiene vómitos ${ }^{9}$. Históricamente se ha descrito una tendencia a presentarse a derecha, pero en algunas series de caso se han reportado una incidencia equitativa entre ambos lados, especialmente en hombres ${ }^{10}$.

\section{Apoyo diagnóstico}

Dentro de las imágenes se describe la TC como la de mayor rendimiento con una sensibilidad mayor al $90 \% \%^{1,7,8,11}$. Si bien es el mejor examen radiológico, puede dar una interpretación equívoca, como se ve en nuestros casos donde fueron 
inicialmente descritos como una hernia femoral. Un saco herniario presente entre los músculos pectíneo y obturador es el signo radiológico que hace diagnóstico ${ }^{12}$. En nuestra serie se puede apreciar ese hallazgo en los tres casos (ver imágenes). Actualmente, la importancia de la TC radica en la posibilidad de diagnosticar esta patología de manera temprana y preoperatoria, factor más relevante en el manejo y pronóstico ${ }^{3}$.

\section{Tratamiento}

Es de manejo quirúrgico.

\section{a) Abordajes}

Abierto transperitoneal, preperitoneal y laparoscópico, son todos abordajes descritos y aceptados para la hernia obturatriz ${ }^{2}$. Si bien la mayoría de los autores coinciden en que el abordaje preferido de hernia obturatriz complicada es la laparotomía media infraumbilical, el abordaje laparoscópico, tanto pre como intraperitoneal, resultan en menor dolor y fiebre en el posoperatorio, menor número de complicaciones pulmonares y menos días de hospitalización ${ }^{13}$. El acceso al agujero obturatriz puede ser complejo en forma laparoscópica dado su anatomía, pero el contexto de pacientes delgados puede aportar cierta ayuda. A pesar de eso, suelen ser abiertas dado el enfoque inicial hacia la obstrucción intestinal presente. En nuestro centro, la cirugía laparoscópica de la hernia abdominal se realiza en forma anecdótica, pero hay gran familiaridad con el manejo abierto preperitoneal. En nuestra serie, dos de los casos fueron operados de este modo, siguiendo la técnica descrita por Nyhus ${ }^{14}$. El abordaje preperitoneal y la cirugía de la hernia abdominal, particularmente la femoral y obturatriz, resulta particularmente cómoda por cuanto brinda una adecuada exposición a la vez que evita la laparotomía, con sus consabidos riesgos (lesiones intestinales, dolor posoperatorio, etc.). Además, en caso de necesitarlo es posible acceder a la cavidad peritoneal por el mismo abordaje, sin mayor problema. Frecuentemente usamos un separador plástico tipo Pelosi para el acceso preperitoneal, logrando buena separación, protección de la herida de eventuales contaminaciones y poco dolor gracias a la dispersión de la presión sobre la herida.

\section{b) Reparación de defecto}

Respecto a la reparación del defecto, está descrita la sutura simple y reparaciones con material biológico o sintético ${ }^{13}$. En general, se prefiere ante, defectos pequeños el uso de suturas simples y ante defectos de mayor tamaño utilizar elementos biológicos o parches sintéticos. En la literatura no se describen grandes diferencias entre las distintas técnicas, independiente del estado de la hernia, posiblemente por lo infrecuente del cuadro ${ }^{7,10,15}$. Dentro de los materiales sintéticos se describen el uso de malla de propileno, tanto por vía preperitoneal como intraperitoneal, y como "cigar roll" o "plug", en general, todas libres de tensión. Se conocen complicaciones del uso de parches sintéticos tales como neuralgias con el uso de plug ${ }^{1}$ y riesgo de infección en ambientes contaminados como peritonitis, necrosis o necesidad de resección ${ }^{13}$. Otras complicaciones como la migración del plug (conocida en hernia inguinal y femoral) no han sido descritas en hernias obturatrices, pero eso también puede ser por un problema de frecuencia.

\section{Pronóstico}

La mayoría de los autores concluyen que el factor más importante en la necesidad de resección y el resultado posoperatorio sería la disminución del tiempo entre el diagnóstico y la cirugía, donde sería de gran utilidad la TC precoz ${ }^{11}$. Dado el tipo de paciente, el diagnóstico tardío (sólo entre $21,5 \%$ y $31,3 \%$ son diagnosticadas correctamente preoperatoriamente) $)^{9}$ y la naturaleza de una patología quirúrgica, es frecuente la morbilidad tanto intrahospitalaria como una vez de alta. Igari et al., publicaron en su serie de casos que el 60-80\% de los pacientes requiere resección intestinal y $56,3 \%$ presenta perforaciones ${ }^{9}$. El tiempo promedio de estadía hospitalaria posterior a la cirugía tiene gran variación en la literatura, principalmente en este tipo de pacientes, que debido a la edad, presentan varias comorbilidades que requieren alargar su estadía. Además, se agrega la morbilidad asociada a la operación, donde la mayoría reporta infección de herida operatoria en el $10 \%$ de los $\operatorname{casos}^{7}$. Clásicamente se reporta un $10 \%$ de recidivas ${ }^{1}$. La mortalidad intrahospitalaria varía de un 12 a $70 \%{ }^{6}$.

\section{Conclusión}

Los 3 casos se condicen con la literatura internacional clásica; las tres pacientes eran del sexo femenino, cercanas a los 80 años, de hábito delgado, y todas con hernias a derecha con historias que, retrospectivamente, parecían clásicas. A pesar de eso, los 3 casos se evaluaron como obstruccio- 
nes intestinales de causa no clara y necesitaron mayor estudio para llegar al diagnóstico preciso. Es posible que la baja frecuencia de esta entidad determine el diagnóstico tardío y los errores en la interpretación de imagenología aquí observados. Con el envejecimiento de nuestra población, es probable que más "little old ladies" se presenten con obstrucciones intestinales de causa no clara, por lo que tener en cuenta diagnósticos como la hernia obturatriz será de utilidad.

\section{Responsabilidades éticas}

Protección de personas y animales. Los autores declaran que para esta investigación no se han realizado experimentos en seres humanos ni en animales.

Confidencialidad de los datos. Los autores declaran que en este artículo no aparecen datos de pacientes.

Conflictos de interés: no hay.

\section{Referencias}

1. Stamatiou D, Skandalakis LJ, Zoras O, Mirilas P, Surg M. Obturator Hernia Revisited: Surgical Anatomy, Embryology, Diagnosis, and Technique of Repair. The American Surgeon 2011;77:1147.

2. Petrie A, Tubbs RS, Matusz P, Shaffer K, Loukas M. Obturator hernia: Anatomy, embryology, diagnosis, and treatment. Clinical Anatomy 2011;24:5629.

3. Nasir BS, Zendejas B, Ali SM, Groenewald CB, Heller SF, Farley DR. Obturator hernia: the Mayo Clinic experience. Hernia 2011;16:315-9.

4. Blach O, Ghosh A. 'Little old lady's hernia' causing small bowel obstruction in a man: a case report with a review of literature on the pathophysiology of obturator hernias. Case Reports 2014; 2014 (nov12 1). Published online 2014 Nov 12. doi: [10.1136/bcr-2014206574]

5. Agarwal D, Sharma G, Agarwal NNK,
Rao J, Garg KM. Interstinal Obstruction Due to Obturator Hernia (A Rarest Presentation). Indian Journal of Surgery 2012;75:388-90.

6. Ijiri R, Kanamaru H, Yokoyama H, Shirakawa M, Hashimoto H, Yoshino G. Obturator hernia: The usefulness of computed tomography in diagnosis. Surgery 1996;119:137-40.

7. Liu J, Zhu Y, Shen Y, Liu S, Wang M, Zhao X, et al. The feasibility of laparoscopic management of incarcerated obturator hernia. Surgical Endoscopy 2016;31:656-60.

8. Mantoo SK, Mak K, Tan TJ. Obturator hernia: diagnosis and treatment in the modern era. Singapore Med J. 2009;50:866-70.

9. Igari K, Ochiai T, Aihara A, Kumagai Y, Iida M, Yamazaki S. Clinical presentation of obturator hernia and review of the literature. Hernia. 2010;14:409-13.

10. Light D, Razi K, Horgan L. Computed tomography in the investigation and management of obturator hernia. Scottish Medical Journal 2016;61:103-5.

11. Sun HP, Chao YP. Preoperative diagnosis and successful laparoscopic treatment of incarcerated obturator hernia. Hernia 2009; 14:203-6.

12. Hodgins N, Cieplucha K, Conneally P, Ghareeb E. Obturator hernia: A case report and review of the literature. International Journal of Surgery Case Reports 2013;4:889-92.

13. Zacharakis E, Purkayastha S, Deeba S, Darzi A. Obturator hernias: A review of the laparoscopic approach. Journal of Minimal Access Surgery 2011;7:201.

14. Nyhus LM. Iliopubic Tract Repair of Inguinal and Femoral Hernia: The Posterior (Preperitoneal) Approach. Surgical Clinics of North America. 1993;73:487-99

15. Ng DCK, Tung KLM, Tang CN, Li MKW. Fifteen-year experience in managing obturator hernia: from open to laparoscopic approach. Hernia 2013;18:381-6. 